\title{
Fragile histidine triad protein expression in nonsmall cell lung cancer and correlation with $\mathrm{Ki}-67$ and with p53
}

\author{
C. Mascaux*, B. Martin*, J.M. Verdebout ${ }^{\#}$, A.P. Meert*, , V. Ninaneף, J.P. Sculier*
}

Fragile histidine triad protein expression in nonsmall cell lung cancer and correlation with Ki-67 and with p53. C. Mascaux, B. Martin, J.M. Verdebout, A.P. Meert, V. Ninane, J.P. Sculier. (C) ERS Journals Ltd 2003.

ABSTRACT: Fragile histidine triad (FHIT) is a tumour suppressor gene, which is altered in a variety of epithelial tumours, including lung cancer. Biochemical and functional pathways of its tumourigenicity are not yet understood. Its role in tumour proliferation is particularly controversial. The purpose of this study was to correlate the expression of FHIT protein in nonsmall cell lung cancer (NSCLC) with tumour proliferation as estimated by Ki-67 antigen and with p53, a suppressor gene.

FHIT, Ki-67 and p53 expression were evaluated by immunohistochemistry in 119 resected NSCLC.

Altogether, 58 tumours were negative (expression $<\mathbf{1 0} \%$ ) for FHIT. The median expression in tumours was $15 \%$ positive cells, in comparison with $100 \%$ in normal matched lung tissue. The expression was as strong as in normal tissue in only 19 cases. FHIT expression was significantly lower in squamous cell carcinoma (SCC) $(5 \%)$ than in adenocarcinoma (ADC) (64\%). The median expression of Ki-67 was $20 \%$ and $69 \%$ of tumours were positives (expression $>10 \%$ ). Ki-67 expression was significantly higher in SCC $(33.3 \%)$ than in ADC $(10 \%)$. The loss of FHIT protein was not correlated with the expression of p53 (median: $7.5 \%, 58 \%$ of positive tumours for a cut-off of $10 \%$ of positive cells) or Ki-67. But percentage of labelled cells for p53 and Ki-67 were significantly correlated.

The results suggest that for fragile histidine triad, the pathway of tumourigenesis is independent of p53 and of tumoural proliferation, as reported previously in vitro. Eur Respir J 2003; 21: 753-758.
Depts of *Internal Medicine and Laboratory of Clinical Investigation and Experimental Oncology and "Pathology, Jules Bordet Institute, "Dept of Chest Medicine, St Peter University Hospital, Free Univerisity of Brussels and ${ }^{\S}$ Fond National de la Recherche Scientifique, Brussels, Belgium.

Correspondence: J.P. Sculier, Service de Médecine, Jules Bordet Institute, 1, rue Héger Bordet, B-1000 Brussels, Belgium.

Fax: 3225343756

E-mail: sculier@bordet.be

Keywords: Fragile histidine triad, Ki-67, lung neoplasms, nonsmall cell lung cancer

Received: October 22002

Accepted after revision: January 102003

This study was supported by La foundation Vésale. B. Martin and A.P Meert received a fellowship from Fonds National pour la Recherche Scientifique (FNRS), Télévie Bruxelles (grant $\mathrm{n}^{\circ}$ 7.4512.98 and $\mathrm{n}^{\circ}$ 3.4579.02).
Fragile histidine triad (FHIT) is a nonclassical tumour suppressor gene encompassing the most fragile site of the human genome, FRA3B. FHIT, located on the short arm of chromosome 3 , at locus 3 p14.2, is homologous to a family of genes characterised by a histidine triad. Abnormalities in this gene are among the most common genetic changes occurring in human cancers. Alteration of FHIT transcript, loss of heterozygocity of the FHIT gene by deletion and loss of the FHIT protein occur in a variety of epithelial tumours including lung cancer [1], head and neck tumours [1, 2], oesophageal, stomach and colon cancers [3], pancreatic carcinoma [4], breast cancer [5], cervical [6] and endometrial [7] carcinoma, renal carcinoma [8], bladder tumours [9] and hepatocellular carcinoma [10].

In lung cancer, loss of FHIT has been documented in $80-100 \%$ of small cell lung cancer and in $40-80 \%$ of nonsmall cell lung cancer (NSCLC). Reduction of FHIT expression is more important in squamous cell carcinoma (SCC) than in adenocarcinoma (ADC) and in smokers than in nonsmokers [1]. Loss of FHIT does not seem to correlate with the prognosis of lung cancer and already occurs in preneoplastic lesions $[1,11,12]$.

Biochemical and functional pathways of FHIT tumourigenicity are not yet understood. FHIT is a diadenosine triphosphate (Ap3A) hydrolase, but the role of this enzyme in suppressing tumours is still unclear. Some recent trials suggest that FHIT could play a role in apoptosis, but this is perhaps not its only biological function. The role of FHIT protein in intrinsic cellular proliferation remains particularly controversial. In vitro, FHIT expression does not seem to alter the growing cell $[13,14]$, but these trials were inconclusive, since the FHIT-expressing clones were selected for constitutive FHIT expression. In contrast, in vivo studies performed in mice [14] suggest that FHIT expression strongly affects the ability of tumoural cells to grow. Based on those considerations, the present study was undertaken to correlate the FHIT protein expression using immunohistochemistry, in a series of resected NSCLC with a tumoural proliferation index (estimated by Ki-67 expression). FHIT expression was also correlated with patient characteristics and another tumour suppressor gene, p53.

\section{Material and methods}

\section{Tissue specimens}

Invasive NSCLC $(n=119)$ was resected by wedge resection, lobectomy or pneumectomy at the Dept of Surgery, St Peter Hospital, January 1993-July 2000. Normal matched lung tissues were also assessed.

\section{Immunohistochemical studies}

Antibodies. For FHIT [14, 15], a polyclonal rabbit antibody anti-GST-FHIT (dilution 1 in 25 , final titration $40 \mu \mathrm{g} \cdot \mathrm{mL}^{-1}$, 
ZR44; Zymed, San Francisco, CA, USA), with cytoplasmic staining, was used. Tumour proliferation was studied with a murine monoclonal antibody (clone MIB-1) to Ki-67 antigen [16], a human recombinant peptide corresponding to a $1002 \mathrm{bp}$ Ki-67 complementary deoxyribonucleic acid (cDNA) fragment (dilution 1 in 50, final titration $1 \mu \mathrm{g} \cdot \mathrm{mL}^{-1}$, Immunotech, Marseille, France). p53 expression was assessed by a mouse monoclonal antibody, MS 186 R7 [17], to suppressor protein Ab-5 (clone DO-7) (dilution 1 in 50, final titration $1.9 \mu \mathrm{g} \cdot \mathrm{mL}^{-1}$, Neomarkers, Union City, CA, USA). Ki-67 and p53 staining were unclear.

Staining. After surgical resection, all tissues were fixed in $10 \%$ neutral buffered formalin and routinely embedded with paraffin. Immunohistochemistry was performed according to a standard streptavidin-biotin-peroxidase complex [18]. Sections of $5 \mu \mathrm{m}$-thickness were cut from paraffin-embedded tissues, deparaffinised in xylene and rehydrated in ethanol. The slides were submitted to antigen retrieval in citric buffer, $0.01 \mathrm{M}$ $\mathrm{pH} 6.0$, consisting in three periods of $5 \mathrm{~min}$ of microwave treatments at $650 \mathrm{~W}$. After recooling at room temperature for $20 \mathrm{~min}$, slides were rinsed twice in tris-hydroxymethylamino-methane $\mathrm{HCl}$ buffer (Tris- $\mathrm{HCl} 0.05 \mathrm{M}, \mathrm{NaCl} 0.09 \%$, $\mathrm{pH} \mathrm{7.6,} \mathrm{Tris-} \mathrm{HCl}$ buffer) for $10 \mathrm{~min}$. All the following steps were followed automatically at $37^{\circ} \mathrm{C}$ in the NexES system (Ventana Medical Systems, Tucson, AZ, USA). The endogenous peroxydases were quenched with hydrogen peroxide for $4 \mathrm{~min}$. The primary antibodies were deposited and incubated for $30 \mathrm{~min}$. The complexes between the FHIT, p53 or Ki-67 proteins and their respective antibodies were fixed with glutaraldehyde and $0.9 \% \mathrm{NaCl}$. The secondary biotinylated antibody was incubated for $8 \mathrm{~min}$. The slides were then stained using a diaminobenzidine tetrahydrochloride (DAB) detection kit (Ventana Medical System), counterstained with haematoxylin and mounted with permount.

Negative controls for the three antibodies were carried out by omitting the primary antibody and substituting normal mouse (for p53 and Ki-67) and rabbit (for FHIT) immunoglobulin for the primary antibody. Positive controls used an internal control, the normal lung epithelial cells for FHIT and, for p53 and Ki-67, external controls from breast tumours well known to be positive for each antibody.

Evaluation of the staining. Observers $(n=3)$ independently evaluated the results of the immunohistochemical staining without any knowledge of the clinical data. The level of positivity was expressed as percentage $(0-100 \%)$ of tumour cells in the total field of a single section showing cytoplasmic (for FHIT) or nuclear (for Ki-67 and p53) staining.

For FHIT, the tumour expression was considered as negative when the expression was $<10 \%$ and positive when $>10 \%$ [1]. Positivity $>90 \%$ was considered to be equal to normal lung tissue. For p53, a tumour was also considered as positive when expression was $>10 \%[19,20]$.

The tumour proliferation index, as estimated by the expression of $\mathrm{Ki}-67$, was expressed as a percentage of stained cells in the total field of a single section and a tumour was considered as positive when $>10 \%$ of cells were immunoreactive $[21,22]$.

\section{Statistical analysis}

Statistical analysis was performed with nonparametric tests, since the distribution of FHIT, Ki-67 and p53 expression was non-normal. The correlation between continuous and dichotomic variables was measured using the Mann-Whitney U-test. Comparisons between the dichotomic variables were performed using corrected Chi-squared tests. The correlation between biological variables, all considered as continuous in order to avoid the problem of a subjective choice of level of positivity, was assessed by Spearman ranks correlation coefficients. Its significance was assessed by testing a null hypothesis of equality to zero of this coefficient. The median values of the distributions of the biological variables were compared according to staging, sex, age, tobacco or histology by Mann-Whitney U-test for dichotomic variables, or using the Kruskal-Wallis test for categorical variables. Survival was measured from the date of surgery. Survival distribution was estimated by the Kaplan-Meier method. Survival comparison was performed by two-sided log-rank tests. The criterion for statistical significance was $\mathrm{p}<0.05$.

\section{Results}

The patient population consisted of 97 males (82\%) and 22 females $(18 \%)$ with a median age of 63 yrs (range 27-86 yrs). Histology, according to the new World Health Organisation 1998 classification [23], was ADC in 52, SCC in 59, adenosquamous cell carcinoma in two and undifferentiated large cell carcinoma in six. Seventeen patients had stage IA disease $(14 \%)$, 52 stage IB (44\%), four stage IIA (3\%), 28 stage IIB $(24 \%), 10$ stage IIIA $(8 \%)$, six stage IIIB $(5 \%)$ and two stage IV $(2 \%$, due to the presence of two tumours in the same lung), according to the 1997 International Staging System [24]. There were 40 active smokers (34\%), 60 former smokers $(50 \%)$ and five nonsmokers (4\%). Information was missing in $14(12 \%)$. Smoking ranged $13-140$ packs-yr with a median of 48 packs-yr.

The median expression of FHIT protein was $15 \%$ (range $0-100 \%$ ) in NSCLC in comparison with $100 \%$ in normal matched lung tissue (fig. 1, d). Among tumours, 58 (48.7\%) were negative for the FHIT protein and 61 (51.3\%) positive. Only $19(16 \%)$ of samples showed an expression as strong as normal tissue. One hundred $(84 \%)$ thus had a reduced or negative FHIT expression. The loss of FHIT protein was not significantly associated with age, sex, stage or tobacco (table 1). Expression (fig. 1, e, f, g, h) was significantly lower $(\mathrm{p}<0.001)$ in SCC (median: $5 \%$, range $0-100 \%)$ than in ADC (median: $64 \%$, range $0-100 \%$ ). The median expression of $\mathrm{Ki}-$ 67 in NSCLC (fig. 1 a, b) was 20\% (range 0-92\%) and was not significantly different according to age, sex, stage or tobacco, but was higher $(\mathrm{p}<0.001)$ in SCC $(33.3 \%$, range $0-90 \%)$ than in $\mathrm{ADC}(10 \%$, range $0-92 \%)$. Using a cut-off of $10 \%, 69 \%$ of the tumours were positive for Ki-67. The median expression of p53 in those tumours (fig. 1c) was 7.5\% (range $0-100 \%$ ) and there was no association with any patient characteristic. For p53, tumours were positive in $48 \%$ of the cases.

If the variable is considered continuous, FHIT expression (table 2) was not correlated with either Ki-67 (globally and according to histology) or p53 expression. However, p53 expression was significantly correlated with the expression of $\mathrm{Ki}-67(\mathrm{r}=0.32, \mathrm{p}=0.0005)$. If only $\mathrm{p} 53$ is considered as a dichotomic variable, the results were the same, showing no significant association between FHIT and p53 expressions $(\mathrm{p}=0.12$, Mann-Whitney U-test) but a significant correlation between p53 and Ki-67 ( $<<0.0001)$. If both FHIT and p53 are considered as dichotomic variables, and if FHIT is evaluated according to positive versus negative (cut-off 10\%), and according to strong versus reduced as compared to normal tissue (cut-off 90\%), there was no significant correlation between FHIT and p53, with p-values of 0.07 and 0.47 , respectively. If FHIT, p53 and $\mathrm{Ki}-67$ were each considered as dichotomic variables, there was also no significant correlation between FHIT and Ki-67 expression $(\mathrm{p}=0.39$ and $\mathrm{p}=0.70$, 

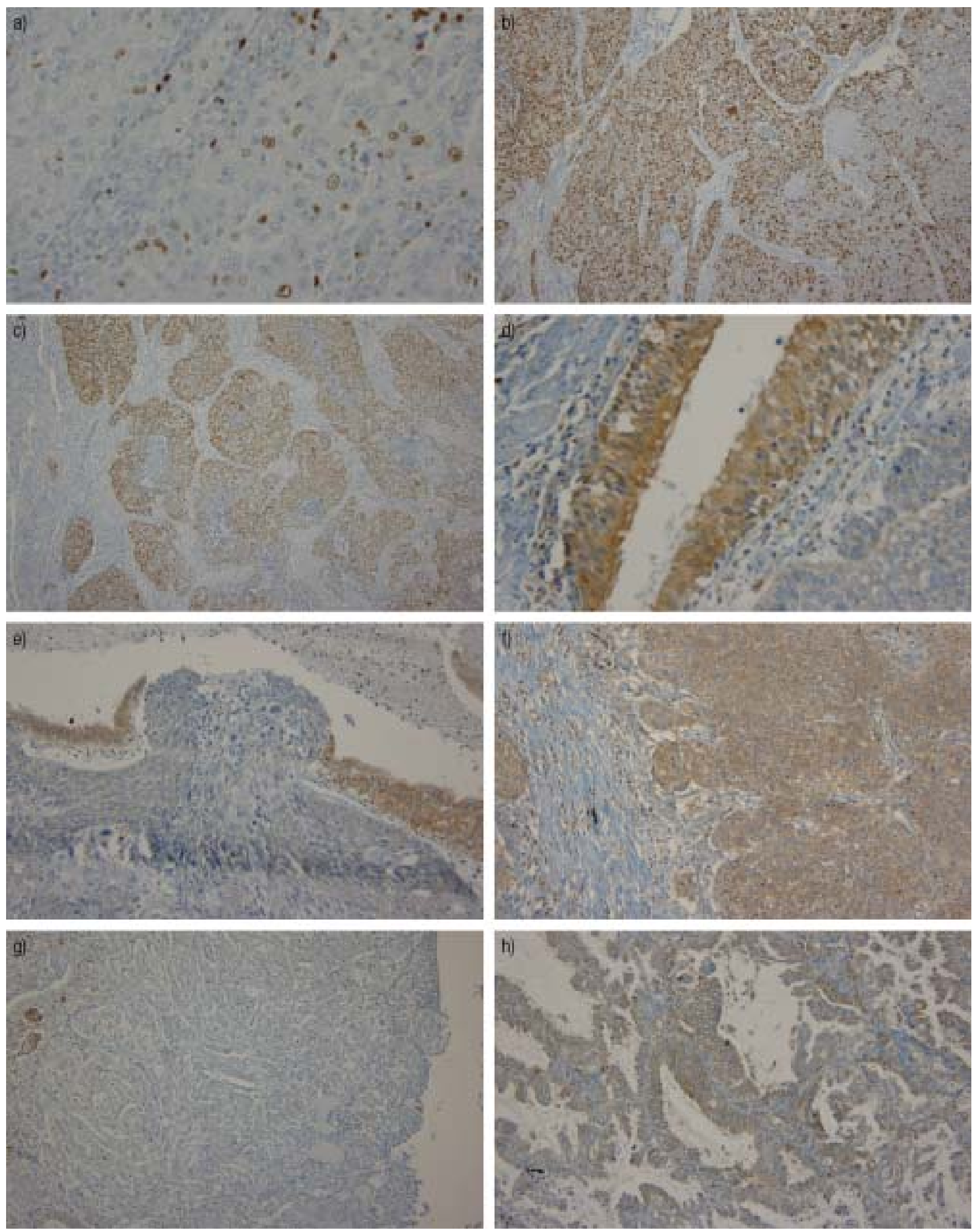

Fig. 1.-Samples with Ki-67, p53 and fragile histidine triad (FHIT) staining by immunohistochemistry. a) Adenocarcinoma (ADC) with a low nuclear staining of Ki-67. b) squamous cell carcinoma (SCC) with a strong nuclear staining of Ki-67. c) SCC with nuclear staining of p53. d) Strong cytoplasmic staining with FHIT in normal epidermal lung cell. e) Low cytoplasmic staining with FHIT in a SCC with internal control contrast. f) Strong cytoplasmic staining with FHIT in a SCC. g) Low cytoplasmic staining with FHIT in an ADC. h) Strong cytoplasmic staining with FHIT in an ADC. 
Table 1.-Correlation between biological variables and patient characteristics

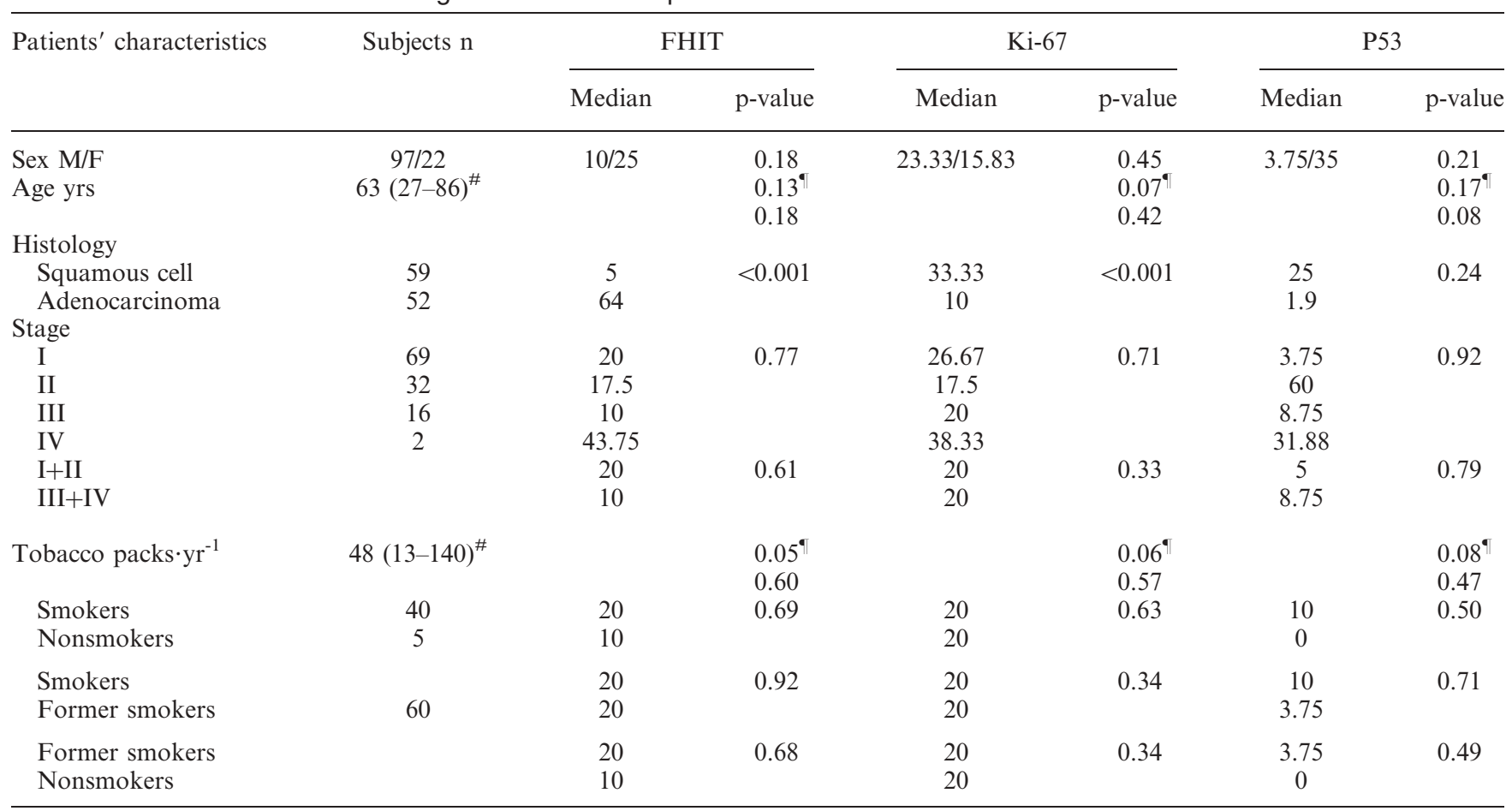

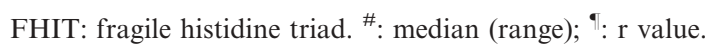

respectively, with a cut-off of $10 \%$ and $90 \%$ for FHIT), but there was still a significant correlation between p53 and Ki-67 expressions $(\mathrm{p}<0.001)$.

The median follow-up was 28 months (range 0.3-109). Among the 119 patients, 51 were dead at the time of analysis. Survival rates at 1 and 3 yrs were $80 \%(95 \%$ confidence interval (CI) $72-88 \%$ ) and 59\% (95\% CI 49-69\%), respectively. The median survival was 53 months. No significant difference in terms of survival distribution according to FHIT expression (fig. 2) was observed. There were 24 and 27 dead patients with positive and negative tumours, respectively, for FHIT expression at the time of analysis.

\section{Discussion}

The results from the present study confirm the reduced expression of FHIT protein in lung cancer with a proportion similar to observations reported by others $[1,11,12]$. It also confirms a higher loss of FHIT in SCC as compared to

Table 2. - Spearman correlation between Fragile Histidine Triad (FHIT), Ki-67 and p53 in nonsmall cell lung cancer

\begin{tabular}{lccc}
\hline & FHIT & Ki-67 & p53 \\
\hline $\begin{array}{l}\text { Expression of markers } \\
\text { median (range) } \%\end{array}$ & $15(0-100)$ & $20(0-92)$ & $7.5(0-100)$ \\
FHIT & & & \\
$\quad$ r & & -0.06 & 0.16 \\
p-value & & 0.54 & 0.10 \\
Ki-67 & -0.06 & & 0.32 \\
$\quad$ r & 0.54 & & 0.0005 \\
p-value & & & \\
p53 & 0.16 & 0.32 & \\
r & 0.10 & 0.0005 & \\
p-value & & & \\
\hline
\end{tabular}

adenocarcinoma $[1,11]$. In addition, the expression of Ki-67 was significantly different according to histology, as reported previously $[25,26]$. No correlation was observed between FHIT expression in NSCLC and tumour proliferation index (estimated by expression of Ki-67), nor with p53 expression, though p53 was significantly correlated with the Ki-67 expression. These results are the same irrespective of the method used to assess the expression of the different markers (as dichotomic variables or as continuous variables) showing that there was no bias due to the choice of the cut-off. This suggests a lack of interference of FHIT with cellular proliferation, as suggested previously by in vitro studies [13, 14], and a biological pathway of FHIT in tumourigenesis independent of tumour proliferation and of $\mathrm{p} 53$. One potential bias has nevertheless to be considered: Ki-67 estimates a measure of cellular proliferation index, but this index is not the only parameter determining tumour proliferation, because

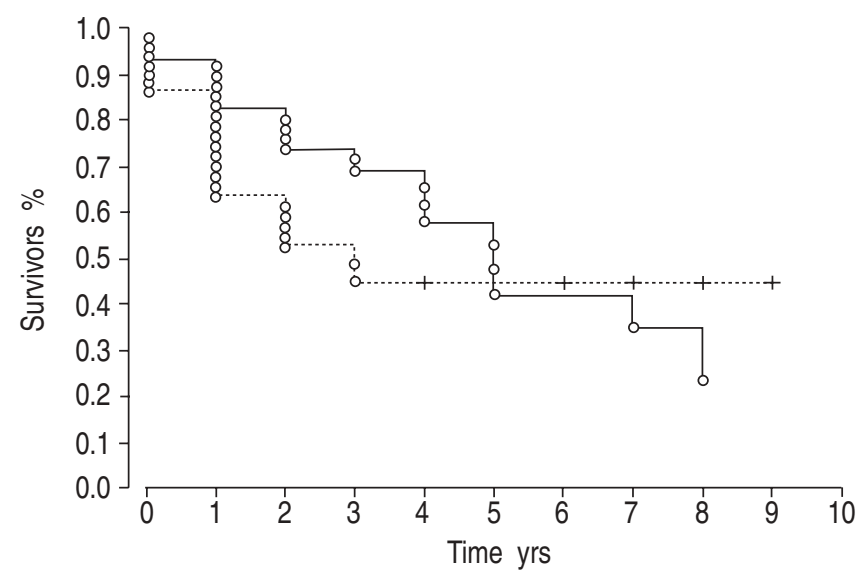

Fig. 2.-Survival according to fragile histidine triad expression. -: positive; .........; negative. 
duration of cellular cycle and cell death rate are other important factors for tumour growing [27]. Ki-67 only evaluates the intrinsic cellular proliferation, not the other two factors determining tumour proliferation. In addition, differences according to tobacco consumption were not demonstrated, though the sample of nonsmokers was too small (five patients) to be conclusive. In terms of survival, preliminary analysis did not detect any significant difference, but a longer duration of follow-up leading to a higher number of events is required to perform a definitive analysis or a larger sample size.

The choice of immunohistochemistry (IHC) to assess expression of FHIT was based on previous publications [15, 27, 28] suggesting that this method was the best way to assess the level of FHIT involvement in a clinical setting because of its high sensitivity and reproducibility as compared with Western-blot analysis or reverse transcriptase polymerase chain reaction. For Ki-67, the clone MIB-1 was chosen as an antibody to estimate the proliferation index, since, in opposition to other factors detecting $\mathrm{Ki}-67$, it can be used with paraffin-embedded tissue [27].

If it is considered that FHIT does not interfere with tumoural proliferation, which other biological pathways could be implicated for this protein? An Ap3A hydrolase activity had never been previously described to suppress tumours. In vivo, the expression of FHIT suppresses tumourigenicity of cell lines transfected in nude mice [14]. The ability to cleave Ap3A is not required for tumour suppression [14], but the binding to Ap3A regulates the activity of FHIT and the FHIT-Ap3A complex could send the tumour-suppressor signal. Interestingly, Ap3A accumulates in cultured cells in response to cellular stress $[29,30]$. The loss of FHIT is a very early event in various tumours, including bronchial preneoplastic lesions [1], breast hyperplasia [31], oral dysplastic lesions [32] and endometrial hyperplasia [7]. Conversley, FHIT does not seem to correlate with prognosis in several trials [1, 11, 12]. These two last points suggest an early role of FHIT in carcinogenesis, in the initiation of tumourigenic process rather than in the progression to the invasive and metastatic disease.

A proapoptotic function of FHIT has recently been reported by in vitro [33-35] and in vivo [33] experiments. Interestingly, apoptosis is associated with a decrease of free Ap3A level in human cultured cells $[19,36]$ suggesting a possible involvement of FHIT in induction of apoptosis through a bound form with Ap3A. Further investigations are required for a better understanding of these potential pathways.

In conclusion, the data from this study suggest that the pathway for fragile histidine triad to suppress tumours seems to be independent of cellular proliferation and different to the p53 pathway. These results need to be confirmed by further analysis, such as cell-cycle profile in fragile histidine triad re-expressing cells. The role of fragile histidine triad in the initiation of cancerogenesis, perhaps at the level of induction of apoptosis, should be investigated in further studies. Common implications in most epithelial tumours and alterations at the earlier stage of tumourigenesis suggest an important role of fragile histidine triad in cancer biology.

Acknowledgements. The authors would like to thank their statistician, M. Paesmans (Data centre, Institut Jules Bordet, Bruxelles, Belgium).

\section{References}

1. Sozzi G, Pastorino U, Moiraghi L, et al. Loss of FHIT in lung cancer and preinvasive bronchial lesions. Cancer Res 1998; 58: 5032-5037.

2. Virgilio L, Shuster M, Gollin SM, et al. FHIT gene alterations in head and neck squamous cell carcinomas. Proc Natl Acad Sci USA 1996; 93: 9770-9775.

3. Ohta M, Inoue H, Cotticelli MG, et al. The human FHIT gene, spanning the chromosome 3p14.2 fragile site and renal cell carcinoma associated translocation breakpoint, is abnormal in digestive tract cancers. Cell 1996; 84: 587-597.

4. Simon B, Bartsch D, Barth P. Frequent abnormalities of the putative tumour suppressor gene FHIT at 3p14.2 in pancreatic carcinoma cell lines. Cancer Res 1998; 58: 1583-1587.

5. Negrini M, Monaco C, Vorechovsky I, et al. The FHIT gene at 3p14.2 is abnormal in breast carcinomas. Cancer Res 1996; 56: 3173-3179.

6. Greenspan DL, Connoly DC, Wu R, et al. Loss of FHIT expression in cervical carcinoma cell lines and primary tumors. Cancer Res 1997; 37: 4692-4698.

7. Ozaki K, Enomoto T, Yoshino K, et al. FHIT alterations in endometrial carcinoma and hyperplasia. Int J Cancer 2000; 85: 306-312.

8. Hadaczek P, Kovatich A, Gronwald J, Lubinski J, Huebner K, McCue PA. Loss or reduction of FHIT expression in renal neoplasias: correlation with histogenic class. Hum Pathol 1999; 11: 1276-1283.

9. Baffa R, Gomella LG, Vecchione A, et al. Loss of FHIT expression in transitional cell carcinoma of the urinary bladder. Am J Pathol 2000; 156: 419-424.

10. Yuan BZ, Keck-Waggoner C, Zimonjic DB, Thorgeirsson SS, Popescu NC. Alteration of the FHIT gene in human hepatocellular carcinoma. Cancer Res 2000; 60: 1049-1053.

11. Geradts J, Fong KM, Zimmerman PV, Minna JD. Loss of FHIT expression in non-small-cell lung cancer: correlation with molecular genetic abnormalities and clinicopathological features. Br J Cancer 2000; 82: 1191-1197.

12. Tseng JE, Kemp BL, Khuri FR, et al. Loss of FHIT is frequent in stage I non-small cell lung cancer and in the lungs of chronic smokers. Cancer Res 1999; 59: 4798-4803.

13. Otterson GA, Xiao G, Geradts J, et al. Protein expression and functional analysis of the FHIT gene in human tumor cells. J Natl Cancer Inst 1998; 90: 426-432.

14. Siprashvili Z, Sozzi G, Barnes LD, et al. Replacement of FHIT in cancer cells suppresses tumorigenicity. Proc Natl Acad Sci USA 1997; 94: 13771-13776.

15. Druck T, Hadaczek P, Fu TB, et al. Structure and expression of the human FHIT gene in normal and tumour cells. Cancer Res 1997; 57: 504-512.

16. Key G, Becker MH, Baron B, et al. New Ki-67-equivalent murine monoclonal antibodies (MIB1-3) generated against bacterially expressed parts of the Ki-67 cDNA containing three 62 base pair repetitive elements encoding for the Ki-67 epitope. Lab Invest 1993; 68: 629-636.

17. Martin B, Verdebout JM, Mascaux C, et al. Expression of p53 in preneoplastic and early neoplastic bronchial lesions. Oncology Reports 2002; 9: 223-229.

18. King G, Payne S, Walker F, Murray GI. A highly sensitive detection method for immunohistochemistry using biotinylated thyramine. J Pathol 1997; 183: 237-241.

19. Nishio M, Koshikawa T, Kuroishi T, et al. Prognostic significance of abnormal p53 accumulation in primary, resected non-small-cell lung cancers. J Clin Oncol 1996; 14: 497-502.

20. Mitsudomi T, Oyama T, Nishida K, et al. P53 nuclear immunostaining and gene mutations in non-small-cell lung cancer and their effects on patient survival. Ann Oncol 1995; 6: Suppl. 3, 9-13.

21. Demarchi LM, Reis MM, Farhat SA, et al. Prognostic values for stromal proportion and PCNA, Ki-67, and p53 proteins in patients with resected adenocarcinoma of the lung. Mod Pathol 2000; 13: 511-520.

22. Carbognani P, Tincani G, Crafa $\mathrm{P}$, et al. Biological markers in non-small cell lung cancer. Retrospective study of 10 year follow-up after surgery. J Cardiovasc Surg 2002; 43: 545-548.

23. Travis WD, Colby TV, Corrin B, Shimosato Y, Brambilla E. World Health Organization. Histological Typing of Lung 
and Pleural Tumors. International Histological Classification of Tumors. 3rd edn. Heidelberg, Springer, 1999.

24. Moutain CF. Revisions in the international system for staging lung cancer. Chest 1997; 111: 1710-1717.

25. Mehdi SA, Etzell JE, Newman NB, Weidner N, Hohman LJ, Graziano SL. Prognostic significance of Ki-67 immunostaining and symptoms in resected stage I and II non-small cell lung cancer. Lung Cancer 1998; 20: 99-108.

26. Nguyen VN, Mirejovsky P, Mirejovsky T, Melinova L, Mandys V. Expression of cyclin D1, Ki-67 and PCNA in non-small cell lung cancer: prognostic significance and comparison with p53 and bcl-2. Acta Histochem 2000; 102: 323-338.

27. Seigneurin $\mathrm{D}$, Guillaud $\mathrm{P}$. L'antigène Ki-67, marqueur du cycle cellulaire et de la proliferation tumorale (Ki-67 antigen, a cell cycle and tumour growth marker). Path Biol 1991; 39: $1020-1028$.

28. Sozzi G, Tornielli S, Tagliabue E, et al. Absence of FHIT protein in primary lung tumors and cell lines with FHIT gene abnormalities. Cancer Res 1997; 57: 5207-5212.

29. Vartanian A, Narovlyansky A, Amchenkova A, Turpaev K, Kisselev L. Interferons induce accumulation of diadenosine triphosphate (Ap3A) in human cultured cells. FEBS Letters 1996; 381: 32-34.

30. Segal E, Le Pecq JB. Relationship between cellular diadenosine 5',5'"'-P1, P4-tetraphosphate level, cell density, cell growth stimulation and toxic stresses. Exp Cell Res 1986; 167: 119126.

31. Dietrich CU, Pandis N, Teixeira MR, et al. Chromosome abnormalities in benign hyperproliferative disorders of epithelial and stromal breast tissue. Int J Cancer 1995; 60: 49-53.

32. Roz L, Wu CL, Porter S, et al. Allelic imbalance on chromosome $3 \mathrm{p}$ in oral dysplastic lesions: an early event in oral carcinogenesis. Cancer Res 1996; 56: 1228-1231.

33. Ji L, Fang B, Yen N, Fong K, Minna JD, Roth JA. Induction of apoptosis and inhibition of tumorigenicity and tumor growth by adenovirus vector-mediated fragile histidine triad (FHIT) gene overexpression. Cancer Res 1999; 59: 3333-3339.

34. Sard L, Accornero P, Tornielli S, et al. The tumorsuppressor gene FHIT is involved in the regulation of apoptosis and in cell cycle control. Proc Natl Acad Sci USA 1999; 96: 8489-8492.

35. Roz L, Gramegna M, Ishii H, Croce CM, Sozzi G. Restoration of fragile histidine triad (FHIT) expression induces apoptosis and suppresses tumorigenicity in lung cancer and cervical cancer cell lines. Proc Natl Acad Sci USA 2002; 99: 3615-3620.

36. Kisselev LL, Justesen J, Wolfson AD, Frolova LY. Diadenosine oligophosphates (Ap(n)A), a novel class of signalling molecules? FEBS Letters 1998; 427: 157-163. 